\title{
Editorial
}

\section{How High the Carbohydrate?}

\author{
G. M. Reaven \\ Stanford University School of Medicine, Geriatric Research, Education and Clinical Center, Veterans Administration Medical Center, \\ Palo Alto, California, USA
}

Accelerated atherogenesis continues to account for considerable morbidity and mortality in patients with diabetes mellitus. Conventional wisdom has it that the incidence of atherosclerosis is increased in proportion to fat consumption, and this generalization has also been applied to patients with diabetes. As a result, there has been a growing tendency to limit dietary fat intake in diabetic patients. This trend has received official sanction from the recent Special Report of the American Diabetes Association's Committee on Food and Nutrition [1], which states that dietary carbohydrate intake for insulin-dependent diabetics "should usually account for $50-60 \%$ of total energy intake." It should be emphasized that no specific suggestions were made concerning the appropriate amount of carbohydrate to be included in the diet of the insulin-independent diabetic. In spite of the fact that no comments were made concerning amount of dietary carbohydrate in this largest group of diabetic patients, it is my perception that many physicians view the Committee's report as license to increase dietary carbohydrate intake in all diabetics. Indeed, this view was given credence when the Chairman of the Committee stated in a recent Editorial that "it was suggested that for most (my underlining) persons the diet should contain $50-60 \%$ carbohydrate [2]".

There is no evidence that restriction of dietary fat will impede the development of atherosclerosis in patients with diabetes. However, information is available concerning the metabolic effects of a high carbohydrate diet, and these observations have been interpreted by some to indicate that such diets have a beneficial effect on carbohydrate and lipid metabolism in patients with diabetes. Coupled with the theoretical argument that low fat diets will protect against atherosclerosis, these studies have provided the impetus for the proposed dietary changes. I do not interpret the available data in quite the same fashion, and will endeavor to explain my concern with the current view that the higher the dietary carbohydrate, the better-off the patient.

One advantage claimed for the use of a high carbohydrate diet is that it enhances insulin sensitivity. Himsworth is usually cited as the discoverer of this phenomenon, and in a series of incisive studies he demonstrated that the plasma glucose response of normal subjects to an oral glucose challenge improved when dietary carbohydrate intake was increased $[3,4]$. However, inspection of the experimental data suggests that a large part of the improvement in glucose tolerance took place when dietary carbohydrate was increased from less than $10 \%$ to $30-40 \%$ of daily caloric intake. Further striking improvement in glucose tolerance did not occur until carbohydrate intake was increased to approximately $60-70 \%$ of daily caloric intake. Himsworth used the conventional oral glucose tolerance test in association with a combined insulin-oral glucose tolerance test to indicate that the improvement in glucose tolerance that follows increased dietary carbohydrate was due to enhanced insulin sensitivity. As before, striking improvement in insulin sensitivity occurred as dietary carbohydrate was increased from very low to moderate amounts, and improvement in glucose tolerance and insulin sensitivity paralleled each other quite closely.

Studies somewhat similar to those of Himsworth have been reported more recently by Brunzell et al. [5] and by Anderson [6]. Brunzell et al. used isocaloric liquid formula diets, containing either $45 \%$ or $85 \%$ carbohydrate for 7-10 days, and compared the responses to an oral glucose challenge following each diet in 7 normal and 5 "chemical" diabetics. The total integrated plasma glucose response area was $8 \%$ lower following the $85 \%$ carbohydrate diet. 
Since the plasma insulin response was not modified by diet, the authors concluded that the improvement in glucose response was due to an increase in insulin sensitivity. Anderson conducted a somewhat similar study on 7 nonobese subjects defined also as having "chemical" diabetes. Oral glucose tolerance tests were performed after one week of isocaloric solid food diets containing $44 \%$ and $75 \%$ of total calories as carbohydrate. Total plasma glucose response during the glucose tolerance test was $11 \%$ lower when performed after the high carbohydrate diet period, and the insulin response was slightly lower. Anderson also concluded that the high carbohydrate diet led to enhanced insulin sensitivity. Given the differences in amount and kind of dietary carbohydrate intake, the $11 \%$ improvement noted by Anderson [6] was remarkably similar to the $8 \%$ improvement reported by Brunzell and colleagues [5].

Thus, high carbohydrate diets seem to be capable of increasing insulin sensitivity, leading to an improvement in plasma glucose response to a glucose challenge. The modest improvement $(8 \%$ and $11 \%)$ noted by Brunzell et al. [5] and Anderson [6] when carbohydrate intake was increased from approximately $45 \%$ to $75-85 \%$ of total calories is consistent with my suggestion that the most dramatic improvement occurs when subjects are changed from low to moderate carbohydrate intakes. However, the crucial question is not whether high carbohydrate diets improve insulin sensitivity, but whether high carbohydrate diets will lead to lower glucose concentrations in patients with diabetes. As is often the case, important physiological questions relevant to the clinical care of diabetics were first addressed by Himsworth. Himsworth and Kerr [7] indicated over 40 years ago that this was an extremely complex issue, and that quite different results occurred in patients classified as being insulin-sensitive (insulindependent) as compared to those defined as being insulin-insensitive (non-insulin-dependent). Thus, "in the case of the sensitive diabetic increase of the carbohydrate content of the diet causes no increase in glycosuria, no rise in the fasting blood sugar level, but produces improvement of sugar tolerance and of sensitivity to insulin. In the case of the insensitive diabetic increase of dietary carbohydrate causes increase in glycosuria, a tendency to high fasting blood sugar levels, impairment of sugar tolerance and little or possibly no increase in sensitivity to insulin."

These results demonstrate that high carbohydrate diets can lead to significant deterioration of glucose tolerance in certain diabetic patients, and it is interesting to note in this context that simply increasing dietary carbohydrate intake by $12-15 \%$ can lead to significant elevations of postprandial glucose con- centrations in normal subjects and patients with "chemical" diabetes $[8,9]$. Similarly, increasing dietary carbohydrate intake from $45 \%$ to $85 \%$ of total calories led to significant deterioration of diabetic control in patients with untreated non-insulin dependent diabetes with fasting hyperglycaemia [10], i. e., mean fasting plasma glucose increased by $15 \mathrm{mg} / \mathrm{dl}$ and mean daily 24 hour glycosuria increased by $56 \mathrm{~g} /$ day. Furthermore, the glycosuria in some patients become so excessive that caloric balance could not be maintained. Thus, these responses seem to parallel the effects noted by Himsworth and Kerr [7]. On the other hand, the $85 \%$ carbohydrate diet did lead to a significant fall (mean $=22 \mathrm{mg} / \mathrm{dl}$ ) in fasting plasma glucose concentration of 9 similar patients studied while they were maintained on either insulin or sulfonylurea therapy. However, it is interesting to note that 24 hour glycosuria was reported to increase by $35 \mathrm{~g} /$ day in the patient with the most dramatic fall in fasting plasma glucose concentration.

Since the above studies [10] of non-insulin dependent diabetics with significant hyperglycaemia were performed with liquid formula diets over a relatively short time-span, it is obviously important to know what happens when similar patients are eating real food. In this regard, the results of three studies appear to be relevant. Thus, Weinsier et al. [11] noted that increasing dietary carbohydrate from approximately $40 \%$ to $55 \%$ did not lead to deterioration in diabetic control in a 20 week out-patient study. However, approximately half of the 18 patients studied had a fasting plasma glucose concentration of less than $125 \mathrm{mg} / \mathrm{dl}$. Furthermore, the only two patients studied with fasting glucose levels in excess of $200 \mathrm{mg} / \mathrm{dl}$ demonstrated a two to threefold increase in 24 hour urine glucose excretion on the $55 \%$ carbohydrate diet. In an in-patient study, Kiehm et al. [12] increased dietary carbohydrate from $43 \%$ to $75 \%$ in 13 diabetic patients treated with either insulin or sulfonylurea agents. Three of the 13 patients did not appear to benefit from the change in diet. However, in the other 10 patients the $75 \%$ carbohydrate diet led to a fall in fasting plasma glucose concentration, associated with a reduction or even cessation of insulin or sulfonylurea treatment. On the other hand, these patients lost weight $(\mathrm{P}<0.001)$ during the high carbohydrate feeding, and the beneficial effect of weight loss in improving glucose tolerance and insulin sensitivity is well-documented [13]. Secondly, the amounts of insulin and sulfonylurea compounds "required" to control plasma glucose levels are not necessarily requirements, but may simply represent the amount a patient is receiving. It is often possible to decrease dose of hypoglycaemic 
agents in such patients, and the study of Kiehm et al. suffers from lack of an after-control, i. e., an attempt to see how patients did when they were placed back on their original diet ( $43 \%$ carbohydrate). Thirdly, the high carbohydrate diet used by Kiehm and associates was very high in fibre, and it is possible that the increased fibre content played an essential role in preventing post-prandial hyperglycaemia when dietary carbohydrate was increased. Perhaps the most persuasive evidence of the beneficial effects of high carbohydrate diets in the treatment of diabetics is the recent work of Simpson et al. [14]. Non-insulin dependent diabetic patients were maintained for six weeks on solid food diets containing approximately $34 \%$ and $61 \%$ of total calories as carbohydrate. A 24 hour plasma glucose profile was performed in 12 patients on each diet. Although glucose levels tended to be lower in general on the $61 \%$ carbohydrate diet, neither mean daily glucose concentration nor Schlichtkrull's $M$ value [15] changed significantly as a function of diet. However, fasting glucose did fall from 7.4 to $6.5 \mathrm{mmol} / \mathrm{l}$ (from approximately 133 to $117 \mathrm{mg} / \mathrm{dl}$ ). Further evidence of the modest improvement in glucose homeostasis was a fall in glycosylated hemoglobin from $9.5 \%$ to $8.3 \%$ on the high carbohydrate diet. On the other hand, it must be emphasized that the majority of these patients were receiving concomitant sulfonylurea therapy, and were wellcontrolled with a mean fasting glucose concentration on the $34 \%$ carbohydrate diet of $133 \mathrm{mg} / \mathrm{dl}$. In order to achieve the modest improvement in glucose homeostasis noted, the patients consumed three meals and three snacks, and approximately $42 \%$ of their total daily caloric intake consisted of wholemeal bread! Although I can't comment upon the practicality of this as a long-term diet in Oxford, I can raise the possibility that long-term compliance with this diet would be difficult to achieve in Palo Alto.

I am aware of only two studies since Himsworth's publications in which the effect of high carbohydrate diets has been studied in patients with insulin-dependent diabetes $[16,17]$. In neither instance was there evidence of a change in either degree of a diabetic control or an increase in insulin requirements. On the other hand, given the erratic nature of plasma glucose levels in insulin-dependent patients [18], the fact that diabetic control and insulin dose do not appear to change with variations in carbohydrate intake may not be too surprising.

Although additional studies of the effects of high carbohydrate diabetic diets may exist, I believe that I have summarized a substantial amount of the evidence relevant to this issue. Given this information, what decisions can we make about the use of high carbohydrate diets in the treatment of diabetes? An obvious observation is that the amount of available information is modest. Furthermore, a large proportion of the studies were carried out over short time periods, and with diets that varied in a lot more than just carbohydrate content. Within these limitations can any generalization be made? I think the answer is a tentative yes. For example, it seems possible that high carbohydrate diets will not substantially change plasma glucose levels and glycosuria in patients with Type II diabetes who are well-controlled on insulin or sulfonylurea compounds. Indeed, if the diet is high in wholemeal bread, there may even be a modest lowering of plasma glucose levels. Similarly, a high carbohydrate diet seems to have no deleterious effect on glucose homeostasis of insulin-treated patients with Type I diabetes. On the other hand, high carbohydrate diets may significantly raise plasma glucose levels in "chemical" diabetics and in poorly controlled and/or untreated patients with non-insulin dependent diabetes.

If diabetic control does not seem to change substantially in many patients on high carbohydrate diets, is there any reason to avoid the high carbohydrate approach as a method to decrease dietary fat in all patients with diabetes? I think there are at least three reasons why this may not be prudent. In the first place, diets which are low in fat will be high in carbohydrate, and it is obvious that such diets could vary tremendously in type of dietary carbohydrate. It is possible that a high carbohydrate diet for many patients will be translated into increased intake of simple sugars, and I am afraid that encouraging patients to eat a high carbohydrate diet can lead to a situation in which $42 \%$ of total daily calories are ingested as Danish pastry, not as wholemeal bread. Under these circumstances, I am not sure that the effect would be as beneficial as the results of Simpson et al. [14].

Secondly, I am concerned about the effects of a high carbohydrate diet in diabetic patients who are untreated and/or not well-controlled. It seems that most patients studied have been relatively well-controlled on either sulfonylurea agents or insulin, i. e., mean fasting plasma glucose ranged from 133 to $144 \mathrm{mg} / \mathrm{dl}[10,11,14]$, and it is possible that the effect of high carbohydrate diets in less well-controlled non-insulin dependent diabetic patients might not be so benign. Individual metabolic data available in two of the studies $[10,11]$ indicate that significant increases in $24 \mathrm{~h}$ urine glucose excretion occurred when the 3 of 27 patients with fasting plasma glucose levels $>200 \mathrm{mg} / \mathrm{dl}$ on the basal diet were switched to a high carbohydrate diet. The problem is obviously of much greater importance in untreated non-insulin 
dependent diabetics, in whom $24 \mathrm{~h}$ glucose excretion increased by $56 \mathrm{~g} \mathrm{[10].}$

Thirdly, there is a real possibility that high carbohydrate-low fat diets will lead to significant elevation of plasma triglyceride (TG) levels in diabetic patients. Hypertriglyceridaemia has been referred to as "the hyperlipemia par excellence of the diabetic [19]." Although more striking in non-insulin dependent diabetes, mean plasma TG levels are also significantly greater than normal in insulin dependent diabetics [20]. There is considerable evidence that fasting hypertriglyceridaemia will result when dietary carbohydrate is increased in patients with glucose intolerance $[9,21]$. Although it is commonly considered to be a temporary phenomenon, the transient nature of carbohydrate-induced increases in TG levels is not supported by a great deal of experimental data. One study [22], which is widely quoted in this regard, has only been published as an abstract in 1965, and simply states "that hypertriglyceridaemia diminished by 7 to 14 days despite continuation of high carbohydrate intake." Indeed, the only documented evidence that high carbohydrate diets do not produce a sustained increase in plasma TG levels is found in a study of South African prisoners by Antonis and Bersohn [23], and the application of these data, (observed in normal subjects with extremely low TG levels) to the situation in diabetic patients with hypertriglyceridaemia is questionable.

The effect of high carbohydrate diets on plasma TG levels in patients with significant fasting hyperglycaemia is less well-definied. High carbohydrate liquid formula diets have been shown to significantly increase plasma TG levels in both Type I [16] and untreated patients with Type II $[21,24]$ diabetes. On the other hand, plasma TG levels have reported to remain stable when Type I [17] and Type II [11] diabetics are fed solid food, high carbohydrate diets. These conflicting results may be due to differences in the kind of food consumed (solid food versus liquid formula), or in the patient populations studied. It might seem reasonable to suggest that this issue should be resolved before advocating the widespread use of high carbohydrate diets. On the other hand, one could argue that hypertriglyceridaemia is not a primary risk factor for the development of coronary disease [25], so why worry. I would submit that this position may be too cavalier. In light of the increased incidence of both hypertriglyceridaemia and coronary artery disease in diabetics [26-32], and the evidence from prospective studies that hypertriglyceridaemia may well be a risk factor [33-38], I would argue that considerable caution should be exercised before embracing a therapeutic program that could accentuate this metabolic problem.
Given these considerations, it seems that the only unequivocal answer to the question of how high the carbohydrate it to say that it shouldn't be too low. Carbohydrate deprivation (in the context of an isocaloric diet) would seem to have no place in the modern treatment of diabetes, and the real unanswered question is whether the carbohydrate content of a diabetic diet should approximate $45 \%$ or $55 \%$ of total calories. Unfortunately, this decision is not a simple function of the putative ability of high carbohydrate diets to increase insulin sensitivity. Indeed, we have demonstrated that the increased sensitivity produced by a high carbohydrate diet can be associated with increased day-long glycaemic excursions [39]. The only way to demonstrate that high carbohydrate diets will not adversely effect diabetic control is to document the effects on plasma and/or urine glucose of such diets in a careful study of a reasonable number of clinically well-defined patients. The diets to be compared must be similar in all but proportion of carbohydrate, and consist of food-stuffs that patients will be able to tolerate chronically. Finally, the patient populations should include both insulin-dependent and non-insulin dependent diabetics, differing in degree of control, and being treated with diet alone, sulfonylureas, or insulin. I don't believe that this information is available, and I don't see how it is possible in its absence to advocate the routine use of high carbohydrate diets in all diabetics.

In conclusion, I believe that the omission of any suggestion by the ADA Committee on Food and Nutrition to increase dietary carbohydrate to $60 \%$ in non-insulin dependent diabetics was prudent, and I intend to follow their advice in this instance. I am afraid I can't say the same thing about their recommendation concerning dietary carbohydrate intake in insulin-dependent diabetics, and I believe that such patients should consume diets that contain 15-20\% of daily calories as protein, with the remainder divided equally between fat (low in cholestrol and saturated fat) and carbohydrate (low in simple sugars). I believe that recommendations leading to major modifications in the kind and amount of carbohydrate and fat in the diabetic diet must be based upon sound experimental data. It is obvious that I have considerable doubts that this criterion has been met in the case of the use of high carbohydrate diets in the treatment of diabetes.

\section{References}

1. Committee of the American Diabetes Association on Food and Nutrition (1979) Special Report - Principles of nutrition and dietary recommendations for individuals with diabetes mellitus. Diabetes Care 2: 520-523 
2. Nuttall FQ (1979) Dietary recommendations for individuals with diabetes mellitus (1979): Summary of report from the Food and Nutrition Committee of the American Diabetes Association. Am J Clin Nutr 33: 1311-1312

3. Himsworth HP (1935) The dietetic factor determining the glucose tolerance and sensitivity to insulin of healthy men. Clin Sci 2: 67-94

4. Himsworth HP, Kerr RB (1939) Insulin and alimentary hyperglycaemia in young normal subjects. Clin Sci 4: 1-17

5. Brunzell JD, Lerner RL, Hazzard WR, Porte D jr, Bierman EL (1971) Improved glucose tolerance with high carbohydrate feeding in mild diabetes. N Engl J Med 284: 521-524

6. Anderson JW (1977) Effect of carbohydrate restriction and high carbohydrate diets in men with chemical diabetes. Am J Clin Nutr 30: 402-408

7. Himsworth HP, Kerr RB (1939) Insulin-sensitive and insulininsensitive types of diabetes mellitus. Clin Sci 4: 119-152

8. Reaven GM, Olefsky JM (1974) Increased plasma glucose and insulin responses to high carbohydrate feedings in normal subjects. J Clin Endocrinol Metab 38: 151-154

9. Ginsberg H, Olefsky JM, Kimmerling G, Crapo P, Reaven GM (1976) Induction of hypertriglyceridemia by a low-fat diet. J Clin Endocrinol Metab 42: 729-735

10. Brunzell JD, Lerner RL, Porte D Jr, Bierman EL (1979) Effect of a fat free, high carbohydrate diet on diabetic subjects with fasting hyperglycemia. Diabetes 23: 138-142

11. Weinsier RL, Seeman A, Herrera G, Assal JP, Soeldner JS, Gleason RE (1974) High- and low-carbohydrate diets in diabetes mellitus: Study of effects on diabetic control, insulin secretion, and blood lipids. Ann Intern Med 8: 332-341

12. Kiehm TG, Anderson JW, Ward K (1976) Beneficial effects of a high carbohydrate, high fiber diet on hyperglycemic diabetic men. Am J Clin Nutr 29: 895-899

13. Olefsky JM, Reaven GM, Farquhar JW (1974) Effects of weight reduction on obesity: studies of carbohydrate and lipid metabolism. J Clin Invest 53: 64-76

14. Simpson RW, Mann JI, Eaton J, Moore RA, Carter R, Hockaday TDR (1979) Improved glucose control in maturity-onset diabetes treated with high-carbohydrate modified fat diet. $\mathrm{Br}$ Med J I: $1753-1756$

15. Schlichtkrull J, Munck O, Jersild M (1965) The M-value, an index of blood-sugar control in diabetes. Acta Med Scand 177: 95-102

16. Bierman EL, Hamlin JT III (1961) The hpyerlipemic effect of a low-fat, high-carbohydrate diet in diabetic subjects. Diabetes 10: $432-437$

17. Stone DB, Connor WD (1963) The prolonged effects of a low cholesterol, high carbohydrate diet upon the serum lipids in diabetic patients. Diabetes 12: 127-132

18. Service FJ, Molnar GD, Rosevear JW, Ackerman E, Gateweed LE, Taylor WF (1970) Mean amplitude of glycemic excursions, a measure of diabetic instability. Diabetes 19 : 644-655

19. Albrink MJ (1974) Dietary and drug treatment of hyperlipidemia in diabetes. Diabetes 23: 913-918

20. Greenfield M, Kolterman O, Olefsky J, Reaven GM (1980) Mechanism of hypertriglyceridaemia in diabetic patients with fasting hyperglycaemia. Diabetologia 18: 441-446

21. Farquhar JW, Frank A, Gross RC, Reaven GM (1966) Glucose, insulin, and triglyceride responses to high and low carbohydrate diets in man. J Clin Invest $45: 1648-1656$

22. Lees RS, Frederickson DA (1965) Carbohydrate induction of hyperlipemia in normal man. Clin Res 13: 327
23. Antonis A, Bersohn J (1961) The influence of diet on serum triglycerides in South African white and Bantu prisoners. Lancet I: $3-9$

24. Reaven GM, Lerner RL, Stern MP, Farquhar JW (1967) Role of insulin in endogenous hypertriglyceridemia. J Clin Invest 46: $1756-1767$

25. Hulley SB, Rosenman RH, Bawol RD, Brand RJ (1980) Special Article - Epidemiology as a guide to clinical decisions: the association between triglyceride and coronary heart disease. N Engl J Med 302: 1383-1389

26. Albrink MJ, Lavietes PH, Man EB (1963) Vascular disease and serum lipids in diabetes mellitus. Observations over 30 years (1931-1961). Ann Intern Med 58: 305-323

27. Reaven G, Calciano A, Cody R, Lucas C, Miller R (1963) Carbohydrate intolerance and hyperlipemia in patients with myocardial infarction without known diabetes mellitus. J Clin Endocrinol Metab 23: 1013-1023

28. Reinheimer W, Bliffen G, McCoy J, Wallace D, Albrink MJ (1967) Weight gain, serum lipids and vascular disease in diabetics. Am J Clin Nutr 20: 986-996

29. Santen RJ, Willis PW, Fajans SS (1972) Atherosclerosis in diabetes mellitus: Correlation with serum lipid levels, adiposity, and serum insulin level. Arch Intern Med 130: 833-843

30. Garcia MJ, McNamara PM, Gordon T, Kannell WB (1974) Morbidity and mortality in diabetics in the Framingham population: sixteen year follow-up study. Diabetes 23: 105-111

31. Reckless JPD, Betteridge DJ, Wu P, Payne B, Galton DJ (1978) High-density and low-density lipoproteins and prevalence of vascular disease in diabetes mellitus. $\mathrm{Br}$ Med J I: 883-886

32. Eaton RP (1979) Lipids and diabetes: The case for treatment of macrovascular disease. Diabetes Care 2: 46-50

33. Brown DF, Kinch SH, Doyle JT (1965) Serum triglycerides in health and in ischemic heart disease. N Engl J Med 273: 947-952

34. Rosenman RH, Friedman M, Straus R, Jenkins CD, Zyzanski SJ, Wurm M (1970) Coronary heart disease in the Western collaborative group study. J Chronic Dis 23: 173-190

35. Kannel WB, Castelli WP, Gordon T, McNamara PM (1971) Serum cholesterol, lipoproteins, and the risk of coronary heart disease: The Framingham study. Ann Intern Med 74: 1-12

36. Carlson LA, Bottiger LE (1972) Ischaemic heart-disease in relation to fasting values of plasma triglycerides and cholesterol: Stockholm prospective study. Lancet I: $865-868$

37. Pelkonen R, Nikkila EA, Koskinen S, Pettinen K, Sarna S (1977) Association of serum lipids and obesity with cardiovascular mortality. Br Med J II: 1185-1187

38. Carlson LA, Bottiger LE, Ahfeldt P-E (1979) Risk factors for myocardial infarction in the Stockholm prospective study: A 14-year follow-up focusing on the role of plasma triglycerides and cholesterol. Acta Med Scand 206: 351-360

39. Kolterman OG, Greenfield M, Reaven GM, Saekow M, Okefsky JM (1979) Effect of a high carbohydrate diet on insulin binding to adipocytes and on insulin action in vivo in man. Diabetes 28: 731-736

Received: August 28, 1980

Gerald M. Reaven, M. D

Stanford University School of Medicine

Geriatric Research, Education and Clinical Center

Veterans Administration Medical Center

Palo Alto, CA 94304, USA 